\title{
Paired associate learning in the rat: Role of hippocampus, medial prefrontal cortex, and parietal cortex
}

\author{
RAYMOND P. KESNER \\ University of Utah, Salt Lake City, Utah
}

\begin{abstract}
Rats were tested for their ability to learn a list of four paired associates (foods paired with spatial locations). In this task, rats display increased levels of correct orienting responses as a function of serial position of the paired associate within the list (temporal order function) when short intertrial intervals are used between paired associates. Furthermore, the rats are more likely to use intramaze rather than extramaze cues. These data suggest that rats utilize a temporal strategy to remember a list of paired associates. After training in this task, new rats received medial prefrontal cortex, hippocampus, parietal cortex, or cortical control lesions and were retested. The data indicate that rats with cortical control lesions continued to display a temporal order function, whereas rats with medial prefrontal cortex lesions showed a reduction in the number of correct orienting responses and failed to demonstrate the temporal order function, suggesting that the medial prefrontal cortex might mediate the selection of temporal strategies. Rats with hippocampal lesions also showed a marked reduction in level of correct orienting responses, but they continue to display a temporal order function, suggesting that these rats can still utilize a temporal strategy, but may have difficulties in remembering the appropriate paired associate items. Rats with parietal lesions did not display any significant changes in their temporal order function.
\end{abstract}

In recent years, there has been a strong interest in determining the neural substrates that mediate temporal coding of information. Two neural regions-namely, the hippocampus and the medial prefrontal cortex - have emerged in both animals and humans as important in representing temporal order information. In rats, support for the involvement of the medial prefrontal cortex in temporal order memory comes from the obseryation that lesions of the medial prefrontal cortex disrupt temporal order memory for spatial location or visual information, as well as frequency memory for spatial locations in combination with the absence of deficits in memory recognition for a single spatial location or visual object (Chiba, Kesner, \& Reynolds, in press; Kesner, 1990; Kesner, 1993; Kesner \& Holbrook, 1987; Kesner \& JacksonSmith, 1992). Similar memory deficits for temporal order or relative recency, as well as judgment of frequency for linguistic and spatial information combined with normal item recognition have been reported in humans with frontal cortex damage (Milner, Corsi, \& Leonard, 1991; Shimamura, Janowsky, \& Squire, 1990; Smith \& Milner, 1988).

In rats, support for an involvement of the hippocampus in temporal order memory comes from the observation

This research was supported by NSF Grant BNS 892-1532 and NIH Grant 2RO1 NS20771-07. The researchers wish to acknowledge Linda Lash for her vital assistance with histological analysis and Pam JacksonSmith for critical reading of the manuscript. Correspondence concerning this article should be addressed to R. P. Kesner, Department of Psychology, University of Utah, Salt Lake City, UT 84112. that lesions of the hippocampus disrupt temporal order for spatial location and visual object information, as well as frequency memory for spatial locations combined with deficits in recognition memory for a single spatial location, but not a single visual object (Kesner, 1990; Kesner, Dakis, \& Bolland, 1993; Kesner \& Novak, 1982). Similar memory deficits for temporal order or relative recency for linguistic and spatial information as well as item recognition for linguistic and spatial information have been reported for humans with hippocampal damage (Chiba et al., in press; Hirst \& Volpe, 1982; Hopkins, Kesner, \& Goldstein, 1993; Kesner, Hopkins, \& Chiba, 1992). Other neural regions, such as the parietal cortex, do not appear to be as important in coding temporal information (Kesner \& Gray, 1989; Kesner \& Jackson-Smith, 1992).

Given that the hippocampus and the medial prefrontal cortex mediate temporal order information, there is a possibility that these two regions might also mediate the selection of temporal strategies. One method that has been employed to measure the use of temporal strategies involves serial pattern learning (Capaldi, 1985; Compton, 1991; Hulse, 1978). In this serial pattern learning paradigm, rats either learn to select specific locations or learn to respond differentially in terms of running speed on the basis of the presentation of fixed sequences of events cued by differential amounts of reinforcement. For example, if rats are given a monotonic descending sequence of reward magnitude, they will respond by running progressively slower to the rewards. Furthermore, lesions of the hippocampus do not disrupt this pattern of responding (Comp- 
ton, 1991). If the same sequence of reward magnitude is distributed across three spatial locations, rats will learn to respond to the three spatial locations on the basis of the order of magnitude of reinforcement. Fimbria-fornix lesioned rats are not impaired in this task unless they are started in the middle of the sequence (Olton, Shapiro, \& Hulse, 1984). Thus, temporal order strategies do not appear to be mediated by the hippocampus. However, it is also possible that, in the above-mentioned tasks, serial order is mostly determined by magnitude of reward, which might be mediated more by the amygdala than by the hippocampus.

Thus, in the present experiment, an attempt was made to devise a task that requires successive presentation of paired stimuli without the need to vary reward magnitude. Rats were trained on a list of paired associates or conditional discriminations (different types of food were paired with different spatial locations). Because the list consisted of only four paired associates, it is possible to utilize a temporal strategy to solve this task. Thus, the purpose of the first series of experiments was to demonstrate that normal rats use a temporal coding strategy for optimal performance and that rats tend to use intramaze rather than extramaze cues to solve this task. The purpose of the second experiment was to examine the role of the medial prefrontal cortex, hippocampus, and parietal cortex in performance of this paired associate task. It was predicted that medial prefrontal cortex lesioned rats would be impaired in the utilization of a temporal strategy, whereas the hippocampus and parietal cortex lesioned rats may not display any deficits.

\section{METHOD}

\section{Subjects}

Fifty-five male Long-Evans rats, weighing 275-350 $\mathrm{g}$ at the start of the experiment, were used as subjects. They were housed individually in standard stainless steel cages with a 14:10-h light:dark cycle. All testing occurred during the light cycle. All rats were placed on food deprivation with ad-lib water and were maintained at $80 \%-85 \%$ of ad-lib weight throughout the experiment.

\section{Apparatus}

The apparatus consisted of an eight-arm maze. The maze consisted of a central hexagonal wooden platform $41 \mathrm{~cm}$ in diameter with eight wooden arms, $61 \mathrm{~cm}$ long and $9 \mathrm{~cm}$ wide, projecting outward from the platform. A food well $(3 \mathrm{~cm}$ in diameter, $1.25 \mathrm{~cm}$ deep) was drilled $0.5 \mathrm{~cm}$ from the end of each arm. This food-well placement prevented subjects from viewing the contents of the well from the center platform. All maze floor surfaces were painted white. Clear Plexiglas retaining walls, $5 \mathrm{~cm}$ high, were placed on both sides along the length of each arm. Clear Plexiglas retaining walls, $20 \mathrm{~cm}$ high, were placed in the $5-\mathrm{cm}$ gaps between arms at the point of attachment between arms and the central platform.

A clear Plexiglas door, $20 \mathrm{~cm}$ high, was placed between each arm and the central platform. Each door was attached by means of a string-and-pulley system to a control board adjacent to the maze room. This system allowed the experimenter to lower (open) and raise (close) each door, thus controlling access to each arm while remaining outside of the maze room.

The maze was centered in a $2.5 \times 1.75 \mathrm{~m}$ room. The surface of the maze was elevated $1 \mathrm{~m}$ above the floor of the maze room and
$1.5 \mathrm{~m}$ below the overhead fluorescent lighting fixture. Several scenic posters of various sizes were placed on walls of the maze room to facilitate the subjects' orientation in space. The center of the apparatus contained a food well that could be rotated automatically, so that four different foods could be presented to the rat for each trial of the four paired associates.

\section{Behavioral Procedure}

The rats were familiarized with the apparatus and were trained to obtain food from the center well and from the end of each of the four arms to be used in the test. After 1 week of pretraining, the rats received from the central platform food well either a Froot Loop cereal that was paired with the arm located to the north, a Coca Puff cereal that was paired with the arm located to the south, a Cookie Crisp cereal that was paired with the arm located to the east, and a Buckwheat cereal that was paired with the arm located to the west. For each rat, a different arbitrary combination of cereal with correct arm location was determined. The rat was first given the opportunity to retrieve and eat a cereal from the center food well, then the rat was given the opportunity to orient in front of any one of the four doors. A correct orienting response was defined as an orientation toward the appropriate door (e.g., north) for the specific food (e.g., Froot Loop) that was presented in the center of the maze. An incorrect orienting response was defined as an orientation toward one of the three inappropriate doors for that specific food. To be scored as an orienting response, the rat had to stand in front of a door (for 2 or $3 \mathrm{sec}$ ), pawing the door or pressing its nose several times against it. Similar to paired associate learning experiments in humans, the rats received feedback concerning the correct arm, so that the correct door leading to the same food reinforcement (e.g., Froot Loop cereal) was opened, regardless of the location of the orienting response. The rat did not have to make a second orienting response, if it made an incorrect orienting response. After the rats ate the food reinforcement, they ran back to the center of the maze.

In Experiment 1, 17 rats received 25 trials, with each trial consisting of a list of four paired associates. An intertrial interval (ITI) of 1-4 sec occurred between paired associate presentations. The ITI was calculated from the time that the rat returned to the center of the maze to the time the next piece of food was presented in the center well. All rats received 2 trials a day for 12 days with $15-\mathrm{sec}$ ITIs between blocks of trials and only 1 trial on the last day. The rats were tested 5 days a week. The first group of subjects $(n=4)$ received the four paired associates in a fixed sequence for each trial (same). The second group of subjects $(n=9)$ received a different sequence of the four paired associates (different) for each trial. The third group of subjects $(n=4)$ received paired associates in a random sequence (random) not at all related to the set of four paired associates. Thus, in the random condition, a rat could receive one paired associate twice within a set of four paired associate presentations within a trial, even though across trials the subject received the same number ( 25 paired associates of each type) as in the different condition. In the same condition, there was a high predictability of the sequence of paired associate presentations based on the temporal sequence. In the different condition, there was an increasing predictability of the sequence of paired associate presentations after the first and subsequent presentations based on the temporal sequence. In the random condition, there was no predictability of the sequence of paired associate presentations.

In Experiment 2, 16 new rats were tested using the different condition, but with either 1-4 sec between each paired associate presentation $(n=9)$ or $15 \mathrm{sec}$ between each paired associate presentation $(n=7)$. The ITI $(15 \mathrm{sec})$ between trials of four paired associate presentations remained the same. The rats again received 25 trials. On the basis of the idea that performance in this task is based in part on memory for positions within the list of paired associates presentations and that this memory decays with time, one 
might expect that performance would be better at short delays than at long delays. Thus, if temporal coding is the critical strategy used to perform the paired associate task, then one would predict a more prominent temporal function across serial position for the shorter ITI relative to that at the longer ITI.

In Experiment 3, the same 14 rats from Experiment 2 were randomly assigned to two groups. The first group $(n=9)$ received five trials a day for 2 days in the different condition, with 1 - to 4-sec ITIs and normal extramaze cues present. The second group $(n=5)$ received the same number of trials, but their maze was surrounded by black paper to form a curtain. This experiment was designed to determine whether the selection of a temporal strategy was dependent on extramaze or intramaze cues.

In Experiment 4, 22 new rats were first given 25 trials, with a list of four paired associates within each trial in the different condition and 1- to 4-sec ITIs between paired associates as well as 15sec ITIs between trials. The rats received 5 trials per day five times a week. At the end of the 25 trials, the rats were randomly divided into four different surgical groups.

In preparation for surgery, the rats were anesthetized with Nembutal ( $50 \mathrm{mg} / \mathrm{kg}$, i.p.) and were given atropine sulfate $(0.2 \mathrm{mg} / \mathrm{kg}$, i.p.) as a prophylactic. Rats in all groups were given a midline incision that was sutured following surgery. The first group of rats $(n=6)$ received bilateral aspiration lesions of the medial prefrontal cortex. The lesions extended from bregma anterior to the frontal pole and included an area $1.5 \mathrm{~mm}$ on each side of midline and $3 \mathrm{~mm}$ below dura. The second group of rats $(n=7)$ received bilateral electrolytic lesions of the hippocampus. Stainless steel electrodes were used to produce a lesion. The electrodes were insulated with Epoxylite, except for a $0.50-\mathrm{mm}$ tip. Direct current (1.2 mA) was applied for $10 \mathrm{sec}$ for each electrode placement. Ten lesions were made in each rat in order to provide complete destruction of the hippocampus. Six of the electrode placements (three in each hemisphere) were intended to destroy the dorsal hippocampus. The other four electrode placements (two in each hemisphere) were intended to destroy the ventral aspect of the hippocampus. The coordinates for the dorsal lesions were $4.6 \mathrm{~mm}$ posterior to bregma, 1, 2.2, and $3.4 \mathrm{~mm}$ lateral to midline, and $2.6 \mathrm{~mm}$ below dura. The coordinates for the ventral lesions were $5.6 \mathrm{~mm}$ posterior to bregma, $5.2 \mathrm{~mm}$ lateral to midline, and 5.6 and $8.1 \mathrm{~mm}$ from the top of the skull. For both the dorsal and ventral hippocampus lesions, just enough skull was drilled away (and a small hole cut in dura) to enable the penetration of the electrode. The third group $(n=5)$, a cortical lesion control, received electrolytic lesions $(1.2 \mathrm{~mA}$ for $10 \mathrm{sec}) 1 \mathrm{~mm}$ below dura and above the dorsal hippocampus and $1,2.2$, and $3.4 \mathrm{~mm}$ lateral to midline. The fourth group $(n=4)$ received parietal cortex lesions. The lesions were made bilaterally by means of aspiration and were intended to destroy parietal areas $5,7,39$, and 40 . In the anterior-posterior plane, the parictal cortex lesion extended from the regions approximately $0.5 \mathrm{~mm}$ anterior to bregma to $3.5 \mathrm{~mm}$ posterior to bregma; in the medial-lateral plane, the lesion began $2 \mathrm{~mm}$ lateral to midline and continued laterally to the rhinal sulcus. All cortical tissue within these dimensions was removed to the lateral ventricles. Care was taken not to damage the underlying hippocampus.

After a 1-week recovery from surgery, the rats were given 25 trials with 5 trials per day five times a week.

\section{Histology}

At the conclusion of the experiment, all lesioned rats were given a lethal i.p. injection of sodium pentobarbital, given .05 cc heparin intracardially, and perfused intracardially with normal saline followed by a $10 \%$ formalin solution. The rats were then decapitated; their brains were removed from the skull and stored in $10 \%$ formalin for 1-2 weeks. The brains were then photographed and sliced, with every fourth slice mounted, stained with cresyl violet, and examined for histological verification of the lesion placement.

\section{RESULTS}

\section{Experiment 1}

The data were analyzed as percent correct orienting responses per serial position in each block of trials for all 25 blocks of trials. Even though the location of errors was noted, the reported data are based on the number of correct orienting responses defined as an orienting response to the location of the correct door. The results for Experiment 1 are shown in Figure 1 as a function of the fixed (same), variable (different), or random presentation of the four paired associates per trial and indicate that there was an improvement in the mean percent correct orienting responses with an increase in the serial position only for the same and different groups, but not for the random group. A two-way analysis of variance (ANOVA) with groups as the between factor and serial position as the within factor revealed a significant group effect $[F(2,14)=$ $12.9, p<.001]$ and a significant serial position effect $[F(3,42)=4.1, p<.01]$. A Newman-Keuls test revealed that the same and different groups displayed more orienting responses than did the random group $(p<.01)$. Furthermore, the groups displayed significantly more correct orienting responses for the fourth serial position than for the first and second serial positions $(p<.01)$ as well as the third serial position $(p<.05)$. There were also more correct orienting responses for the third serial position than for the first serial position $(p<.01)$.

\section{Experiment 2}

The results for Experment 2 are shown in Figure 2 and show the effects of short (no delay) and long (delay) ITI on mean percent correct orienting responses as a function of serial position. The data indicate that there is an increasing improvement in performance as a function of serial position for the no-delay condition relative to the

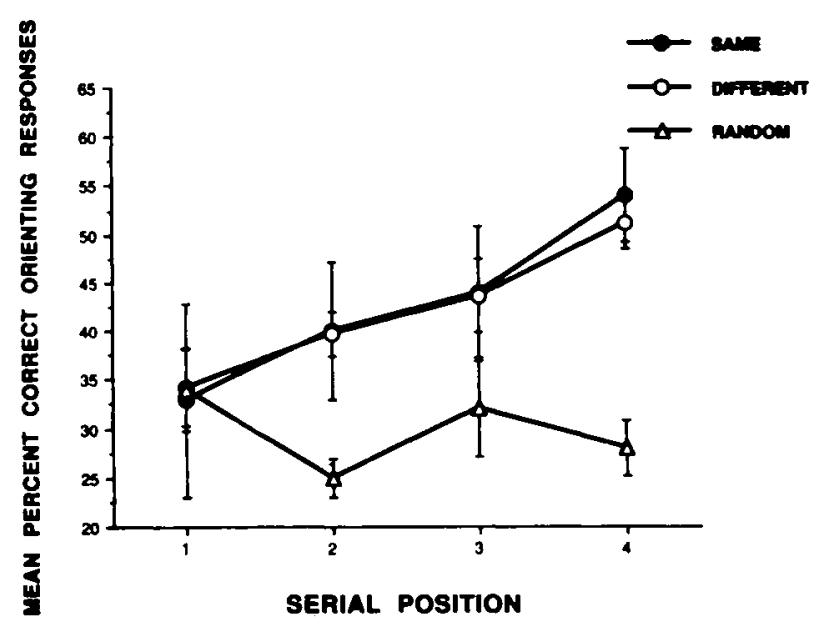

Figure 1. Mean percent correct orienting responses and standard error of the mean as a function of serial position based on fixed (SAME), variable (DIFFERENT), or RANDOM presentations of the four paired associates per trial. 


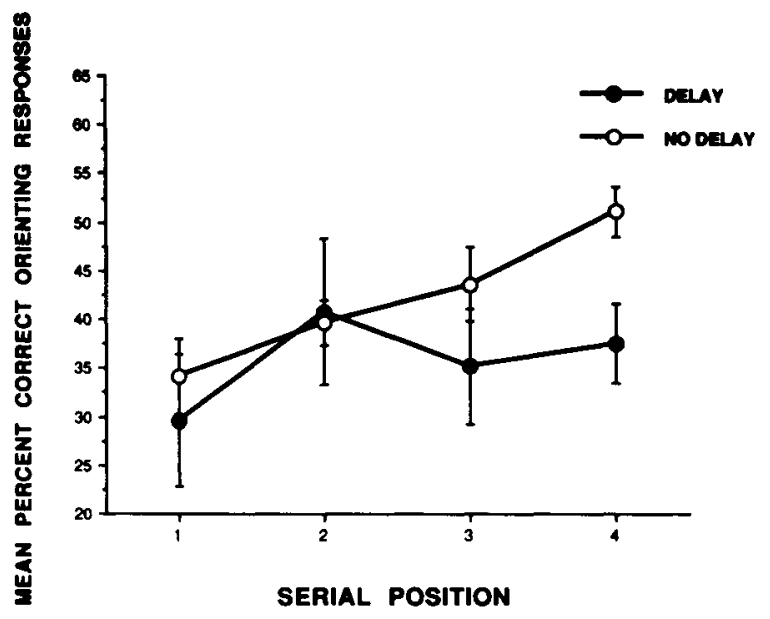

Figure 2. Mean percent correct orienting responses and standard error of the mean as a function of serial position based on short (1-4 sec, NO DELAY) versus long (15 sec, DELAY) intertrial intervals between trials.

Table 1

Mean Percent Retrospective Errors

\begin{tabular}{lccc}
\hline & \multicolumn{3}{c}{ Position From Target } \\
\cline { 2 - 4 } & -1 & -2 & -3 \\
\hline Delay & 13.7 & 23.7 & 28.0 \\
No delay & 3.7 & 16.0 & 24.9 \\
\hline
\end{tabular}

delay condition. A two-way ANOVA with groups as the between factor and serial position as the within factor revealed a significant delay effect $[F(1,12)=4.9, p<.05]$ and a significant serial position effect $[F(3,36)=3.3$, $p<.03]$. With respect to an error analysis of paired associates that preceded the target paired associate, it can be seen in Table 1 that there were more retrospective errors for the immediately preceding paired associate in the long-delay group relative to the short-delay group. An ANOVA with delay as the between factor and position from target as the within factor revealed that there was a significant delay effect $[F(1,14)=18.8, p<.0007]$ and a significant position-from-target effect $[F(2,28)=$ $25.1, p<.0001]$, but the interaction was not significant. Thus, it appears that memory for the preceding paired associate may play an important role in determining the monotonic increasing serial position function, and it appears that there is likely to be faster forgetting of each paired associate with longer delays.

\section{Experiment 3}

The results for Experiment 3 are shown in Figure 3 and show the effect of elimination of extramaze cues on mean percent correct orienting responses as a function of serial position. The data indicate that there is an increasing improvement in performance as a function of serial position even in the absence of extramaze cues. A two-way ANOVA with groups (cues or no cues) as the between factor and serial position as the within factor revealed a nonsignificant effect for cues versus no cues $[F(1,12)=$ $0.025, p=.88$ ], a significant serial position effect $[F(3,36)=20.4, p<.0001]$, and a significant cues versus no cues $\times$ serial position interaction $[F(3,36)=$ $4.4, p<.01]$.

\section{Experiment 4}

The results for Experiment 4 are shown in Figures 4, 5,6 , and 7 and indicate that there is a deficit in mean percent correct orienting responses as a function of serial position following hippocampus or medial prefrontal cortex lesions, but no deficit for parietal cortex or control lesions. A three-way ANOVA with lesion group as the between factor and pre/post and serial position as the within factors revealed a significant lesion group effect $[F(3,18)=5.3, p<.009]$, a significant pre/post effect

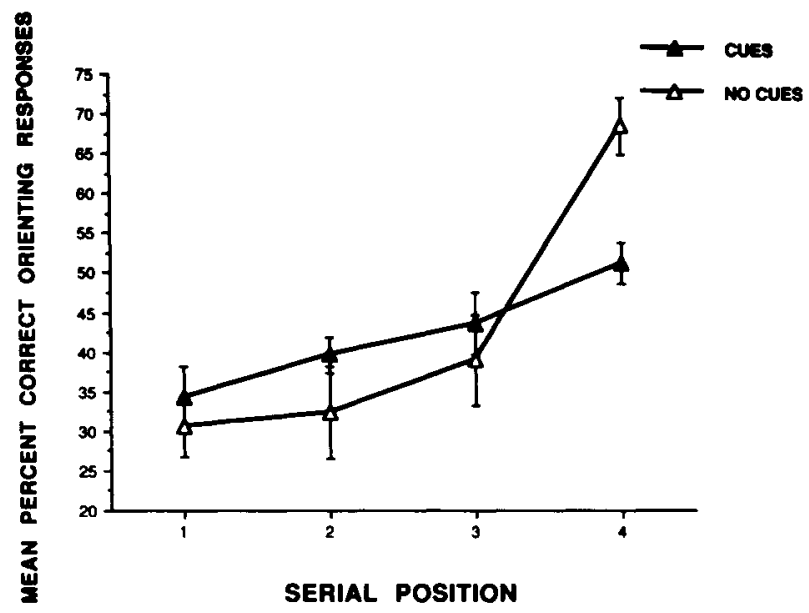

Figure 3. Mean percent correct orienting responses as a function of serial position based on the presence (CUES) versus absence (NO CUES) of extramaze cues.

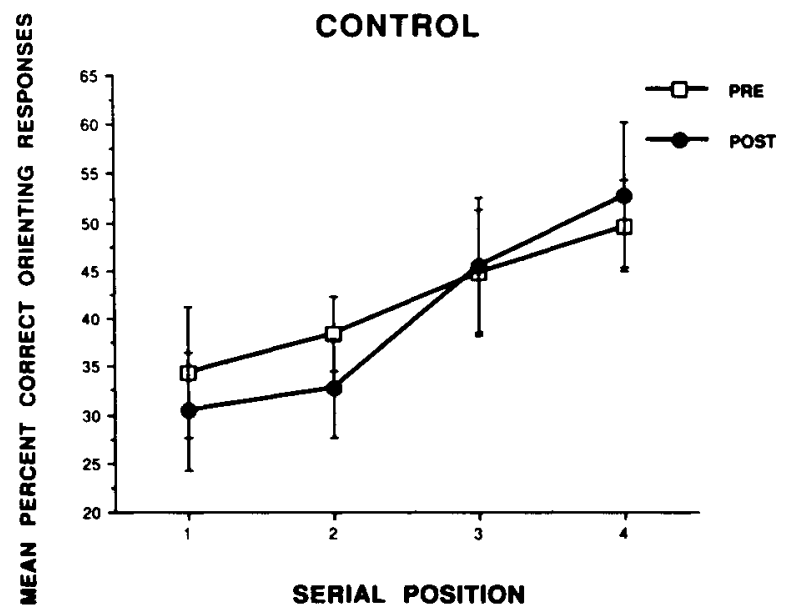

Figure 4. Mean percent correct orienting responses and standard error of the mean as a function of serial position based on pre- and postsurgery performance for the control group. 


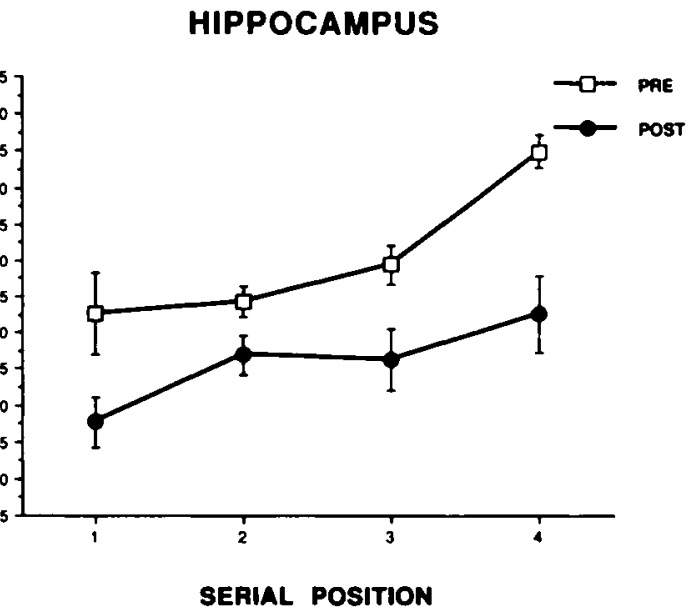

Figure 5. Mean percent correct orienting responses and standard error of the mean as a function of serial position based on pre- and postsurgery performance for the hippocampus lesioned group.

$[F(3,54)=13.7, p<.0001]$, a significant lesion group $\times \mathrm{pre} /$ post interaction effect $[F(3,18)=4.2, p<.02]$, and a significant lesion group $\times$ pre/post $\times$ serial position interaction effect $[F(9,54)=2.1, p<.05]$. Furthermore, Newman-Keuls tests revealed that there were no significant pre/post differences for the control group, but a significant pre/post difference with fewer correct orienting responses after the lesion for all serial positions for the hippocampus group $(p<.01)$. There were no significant pre/post differences for the parietal cortex group, with the exception of a significant reduction in correct orienting responses after the lesion for only the first serial position $(p<.01)$. There were significantly fewer orienting responses postsurgery relative to presurgery for the third and fourth serial position for the medial prefrontal cortex group $(p<.01)$.

With respect to serial position effects, there were more correct orienting responses for the last serial position relative to the first, second, or third serial positions for both pre- and postsurgery conditions for the control group as well as parietal cortex group $(p<.01)$. In the hippocampal group, there were significantly more correct orienting responses for the last serial position relative to the first serial position for both pre- and postsurgery conditions $(p<.05)$. Finally, for the medial prefrontal cortex group, there were significantly fewer correct orienting responses for the fourth serial position relative to the first serial position after surgery compared with the presence of more correct orienting responses before surgery $(p<.05)$. After surgery, with the exception of the medial prefrontal cortex group, all groups displayed increases in orienting responses across the sequential presentation of the four paired associates. With respect to an error analysis of paired associates that preceded the target paired associate, it can be seen in Table 2 that there were more retrospective errors for the medial prefrontal cortex and hippocampus lesioned rats compared with pre- surgery error levels or for rats with parietal cortex or cortical control lesions.

An ANOVA with lesion group as the between factor and pre/post and position from target as within factors revealed a significant lesion effect $[F(3,18)=6.0$, $p<.005]$, a significant pre/post effect $[F(1,18)=10.8$, $p<.004$ ], a significant position-from-target effect $[F(2,36)=114.4, p<.0001]$, and a significant pre/post $x$ lesion interaction $[F(3,18)=5.0, p<.006]$. A subsequent Newman-Keuls analysis on the pre/post $\times$ lesion interaction revealed that the rats with medial prefrontal cortex or hippocampal lesions made significantly more errors than did the cortical control and parietal cortex lesioned rats $(p<.01)$ and made more errors relative

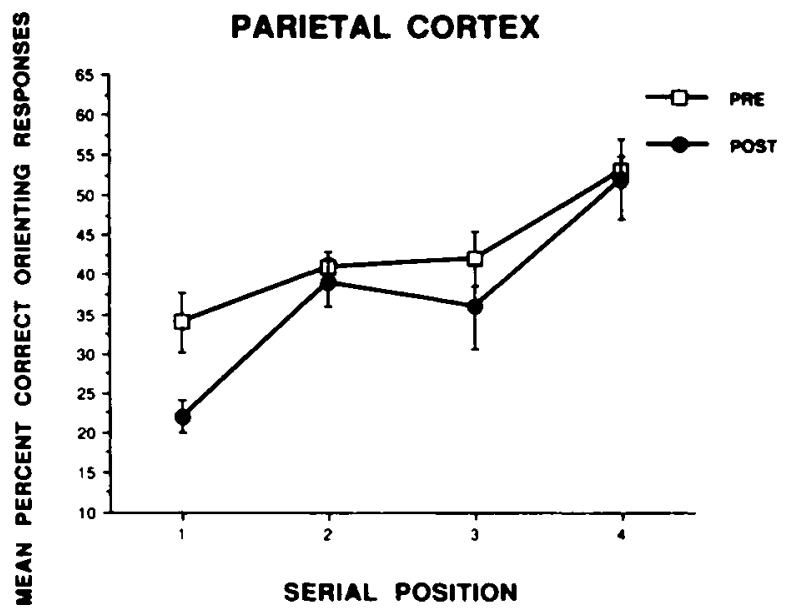

Figure 6. Mean percent correct orienting responses and standard error of the mean as a function of serial position based on pre- and postsurgery performance for the parietal cortex lesioned group.

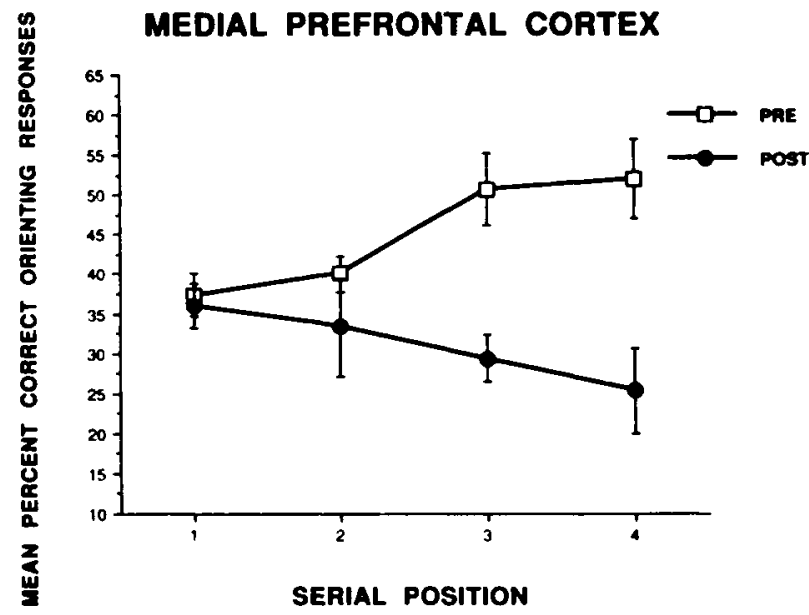

Figure 7. Mean percent correct orienting responses and standard error of the mean as a function of serial position based on pre- and postsurgery performance for the medial prefrontal cortex lesioned group. 
Table 2

Mean Percent Retrospective Errors

\begin{tabular}{lrrrrrrr}
\hline & \multicolumn{4}{c}{ Position from Target } \\
\cline { 2 - 4 } \multicolumn{1}{c}{ Groups } & -1 & -2 & -3 & -1 & -2 & -3 \\
\cline { 2 - 7 } \multicolumn{1}{c}{ Presurgery } & & \multicolumn{3}{c}{ Postsurgery } \\
\hline Control & 4.3 & 20.0 & 20.8 & 3.5 & 14.0 & 20.0 \\
Medial Prefrontal Cortex & 6.7 & 17.7 & 22.0 & 11.1 & 26.7 & 32.0 \\
Hippocampus & 2.7 & 13.4 & 25.1 & 6.6 & 26.6 & 33.6 \\
Parietal Cortex & 1.7 & 19.0 & 22.0 & 9.3 & 18.5 & 18.0 \\
\hline
\end{tabular}

to presurgery levels $(p<.01)$. All groups increased their retrospective error level with increased distance from the target paired associate.

\section{Histology}

The parietal cortex lesions were complete and consistent across subjects. A representative lesion is presented in Figure 8. Microscopic examination of the brains revealed cell degeneration in the lateral posterior, posterior,
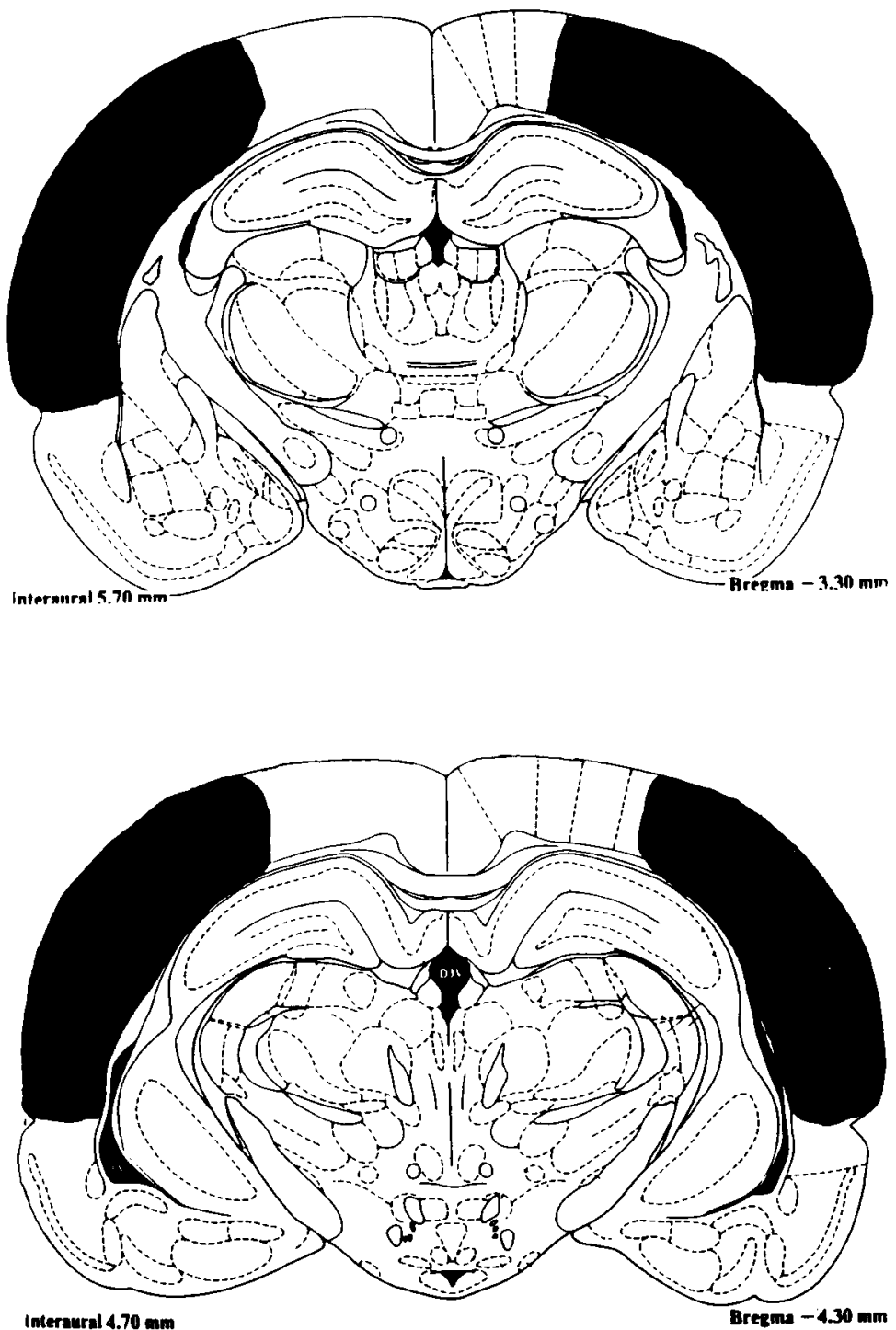

Figure 8. A representative example of a parietal cortex lesion. 
and ventral posterior nuclei of thalamus, which indicated that the lesion destroyed the intended parietal areas. There was no evidence of degeneration in the LGN. The hippocampal lesions also tended to be complete. Coronal sections from a representative lesion are presented in Figure 9. The dorsal hippocampus destruction was, in all rats, total. There was a small degree of sparing in the ventral hippocampus in most of the rats. A representative cortical control lesion is shown in Figure 10. There was no damage to the hippocampus in any of the subjects. A representative medial prefrontal cortex lesion is shown in Figure 11. The damage, based on Groenewegen's (1988) division of medial prefrontal cortex, included medial precentral, dorsal anterior cingulate, and at times prelimbic and lateral precentral area regions.

\section{DISCUSSION}

The results indicate that there is an improvement in correct performance as a function of serial order of presentation of the same or different set of paired associates, but no improvement for the random set of paired associates (Experiment 1). Furthermore, this temporal order function is dependent on the appropriate (e.g., short) temporal separation between the presentation of each set of paired associates (Experiment 2). Thus, repeated lists of paired associates or conditional discriminations can have an inherent property of triggering the use of a temporal coding strategy to learn and remember paired associates within the task. In the present task, this temporal code was evidenced by an increase in correct performance as
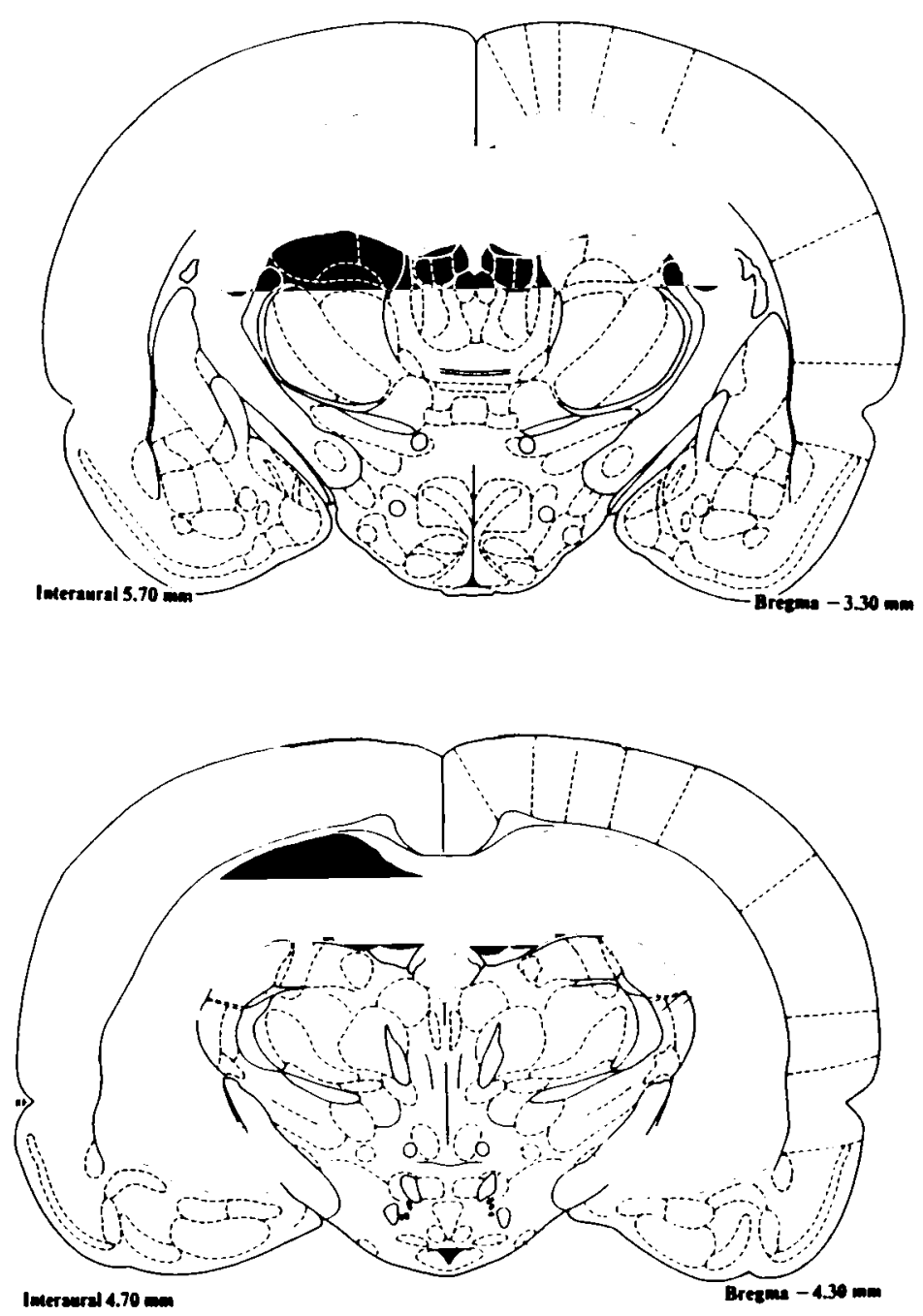

Figure 9. A representative example of a hippocampus lesion. 


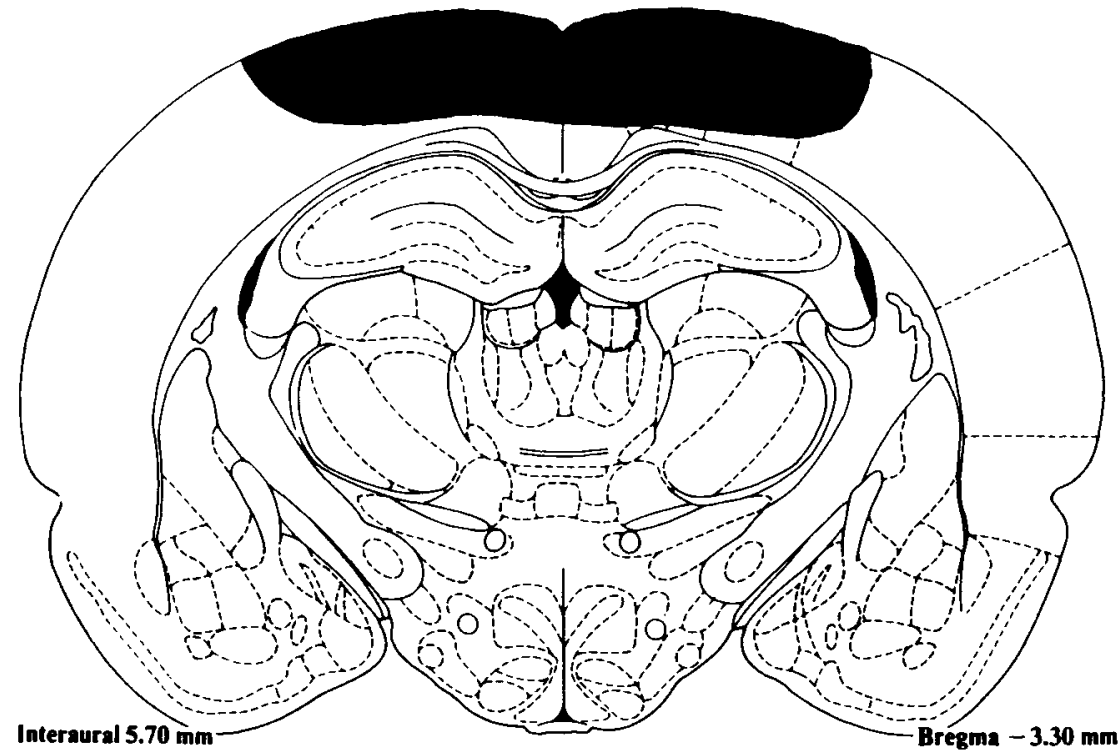

Figure 10. A representative example of a cortical control lesion.

a function of serial order of presentation. Also, in part, this temporal code appears to be based on memory for the preceding paired associate within the list, in that very few errors were made to the immediately preceding paired associate within the list (Tables 1 and 2). It also appears that in this task the rat can succeed by utilizing intramaze rather than extramaze cues on the basis of a lack of interference effects when extramaze cues were removed (Experiment 3).

With respect to the lesion experiment (Experiment 4), the results indicate that control rats continue to display a monotonic increasing function in the number of correct orienting responses as a function of serial order of presentation, implying that control rats continue to use a temporal coding strategy to solve the task. The results also indicate that rats with medial prefrontal cortex lesions are impaired in this task, as indicated by a decrease rather than an increase in the number of correct orienting responses as a function of serial order of presentation. They performed well after surgery for the first two serial presentations. This suggests that there is no problem in displaying orienting responses, but that there is an impairment in the utilization of an appropriate temporal coding strategy, so that improvements in performance could not occur. Furthermore, these rats made more retrospective errors than did control rats or presurgery rats, suggesting a problem in remembering previously experienced paired associates. Thus, these data are consistent with the idea that the medial prefrontal cortex plays an important role in mediating temporal attribute information, either in the form of memory for temporal order or for the utilization of temporal strategies. The data are consistent with the finding of temporal order memory or relative recency deficits in rats and humans following prefrontal cortex damage (Kesner \& Holbrook, 1987; Milner et al., 1991; Shimamura et al., 1990). The data are also consistent with deficits found in monkeys and humans with frontal lobe lesions in visual-spatial, visual-motor, or visual-visual paired associate or conditional discrimination learning tasks (Petrides, 1991). The procedures used in the Petrides studies parallel those used in the present study. It is possible, even though not reported, that the above-mentioned tasks also involved important temporal coding strategies.

The results indicated that parietal cortex lesioned rats continue to display normal orienting responses as a function of serial order of presentation. Thus, these rats were able to use a temporal coding strategy to solve the task. On the basis of the retrospective error analysis, they appeared to have relatively normal memory for previously experienced paired associates. It was not expected that there would be a deficit following parietal cortex lesions, because the task can be solved on the basis of intramaze cues, suggesting a more egocentric coding strategy. Previous research has indicated that parietal cortex lesions in rats do not markedly affect temporal order recognition memory and do not affect learning of an egocentric spatial task (Kesner, Farnsworth, \& DiMattia, 1989; Kesner \& Gray, 1989). Rather, the deficits are more likely to occur in allocentric spatial tasks, such as the water maze or the dry-land version of the water maze (DiMattia \& Kesner, 1988; Kesner et al., 1989).

In contrast, hippocampal lesioned rats display a marked reduction in level of correct orienting responses, but they continue to display an increasing serial order function. 


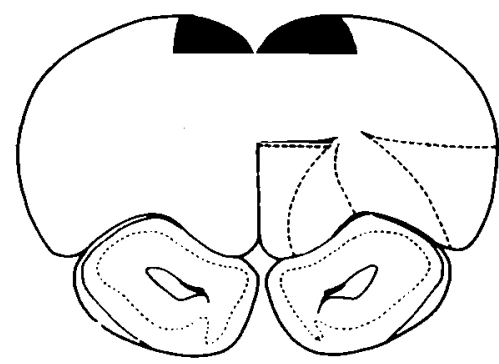

Interaural $13.20 \mathrm{~mm}$

Bregma $4.20 \mathrm{~mm}$

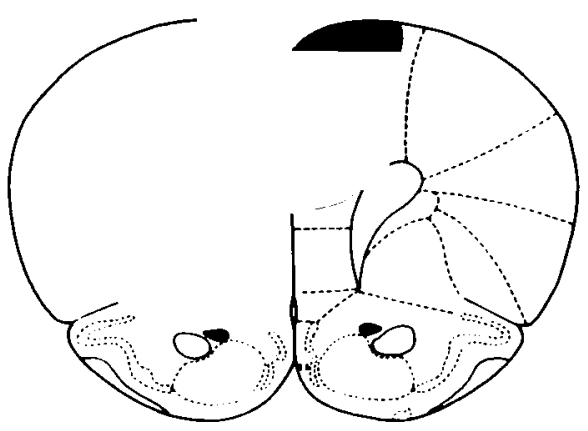

Interaural $12.20 \mathrm{~mm}$

Bregma $3.20 \mathrm{~mm}$

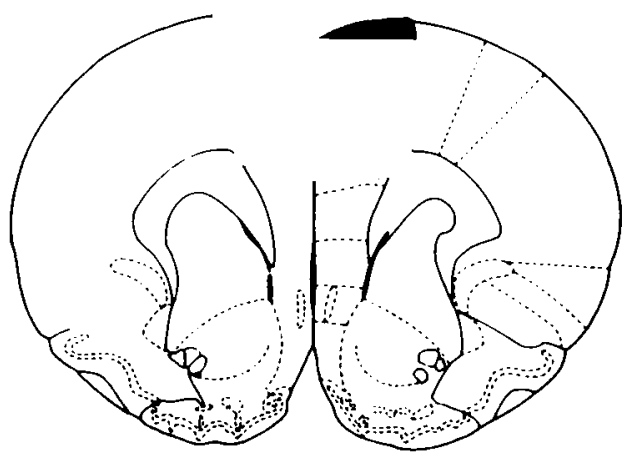

Interaural $11.20 \mathrm{~mm}$

Bregma $2.20 \mathrm{~mm}$

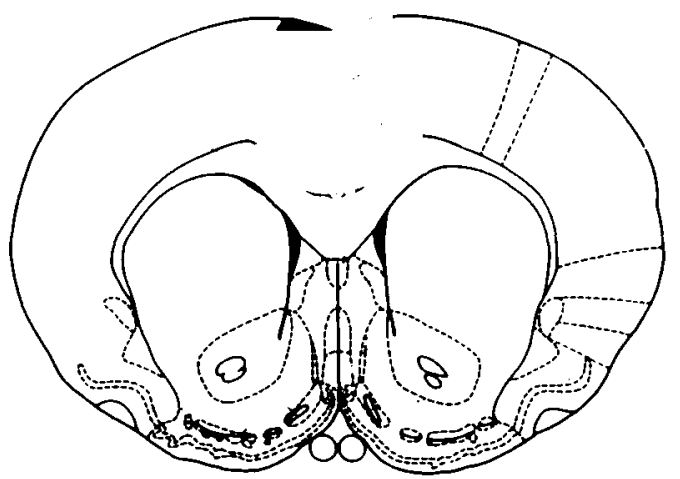

Interaural $10.20 \mathrm{~mm}$

Bregma $1.20 \mathrm{~mm}$

Figure 11. A representative example of a medial prefrontal cortex lesion.
Furthermore, on the basis of the retrospective error analysis, hippocampal lesioned rats made more errors than did control rats or presurgery rats. This suggests that hippocampal lesioned rats can still utilize a temporal strategy, but that they have an overall reduction in learning to select or remembering the appropriate paired associate items. Thus, there appears to be a dissociation between the medial prefrontal cortex and hippocampus in that the impairment for medial prefrontal cortex damaged subjects may be due to the inability to use appropriate temporal strategies and that the impairment for the hippocampus may be due to the inability to learn or benefit from previous exposures to the list of paired associates. This interpretation is consistent with Winocur $(1991,1992)$, who has also suggested that prefrontal cortex, but not hippocampal, damaged rats have difficulty learning rules; however, in this case, learning and performance of a conditional discrimination (visual-motor response) or a matching-to-sample of visual cues was measured. In both tasks, rats with medial prefrontal cortex lesions had a marked impairment in learning the task even at 0 -sec delay, whereas hippocampus lesioned rats learned the task at the 0 -sec delay but were impaired only at long delays. There is also a dissociation between medial prefrontal cortex or hippocampus and parietal cortex in that no deficits were observed for parietal cortex lesioned subjects.

In summary, to the extent that this paired associate task facilitated the use of temporal strategies, the medial prefrontal cortex can be shown to play a very important role.

\section{REFERENCES}

CAPAldi, E. J. (1985). Anticipation and remote associations: A configural approach. Journal of Experimental Psychology: Leaming. Memory, \& Cognition, 11, 444-449.

Chiba, A. A., Kesner, R. P., Reynolds, A. M. (in press). Memory for spatial location as a function of temporal lag in rats: Role of hippocampus and medial prefrontal cortex. Behavioral \& Neural Biology.

Compton, D. M. (1991). Serial learning: A review of the behavioral and physiological research with the rat. Neuroscience \& Biobehavioral Reviews, 15, 363-374.

DiMatria, B. V., \& Kesner, R. P. (1988). Spatial cognitive maps: Differential role of parietal cortex and hippocampal formation. $B e$ havioral Neuroscience, 102, 471-480.

Groenewegen, H. J. (1988). Organization of the afferent connections of the mediodorsal thalamic nucleus in the rat, related to the mediodorsal-prefrontal topography. Neuroscience, 24, 379-431.

HIRST, W., \& VolPE, B. (1982). Temporal order judgements with amnesia. Brain \& Language, 1, 294-306.

Hopkins, R. O., Kesner, R. P., GoldSTEIN, M. (1993). ltem and order recognition memory for words, pictures, spatial locations, abstract pictures, and motor responses in subjects with hypoxic brain injury. Manuscript submitted for publication.

HulSE, S. H. (1978). Cognitive structures and serial pattern learning by rats. In S. H. Hulse, H. Fowler, \& W. K. Honig (Eds.), Cognitive processes in animal behavior (pp. 311-340). Hillsdale, NJ: Erlbaum.

KesNer, R. P. (1990). Memory for frequency in rats: Role of the hippocampus and medial prefrontal cortex. Behavioral \& Neural Biology, $53,402-410$.

KesNer, R. P. (1993). [The effects of medial prefrontal cortex lesions on memory for temporal order of visual objects in the rat]. Unpublished raw data. 
Kesner, R. P., Dakis, M., \& Bolland, B. (1993). Memory for objects, spatial locations and motor responses: Triple dissociations among the hippocampus, caudate nucleus and extrastriate visual cortex. Experimental Brain Research, 93, 462-470.

Kesner, R. P., Farnsworth, G., \& DiMattia, B. V. (1989). Doubledissociation of egocentric and allocentric space following medial prefrontal and parietal cortex lesions in the rat. Behavioral Neuroscience, 103, 956-961.

Kesner, R. P., \& Gray, M. L. (1989). Dissociation of item and order memory following parietal cortex lesions in the rat. Behavioral Neuroscience, 103, 907-910

KesNer, R. P., \& HolbroOK, T. (1987). Dissociation of item and order spatial memory in rats following medial prefrontal cortex lesions. Neuropsychologia, 25, 653-664.

Kesner, R. P., Hopkins, R. O., \& ChibA, A. A. (1992). Learning and memory in humans with an emphasis on the role of the hippocampus. In L. Squire \& N. Butters (Eds.), Neuropsychology of memory (2nd ed., pp. 106-121). New York: Guilford.

Kesner, R. P., \& Jackson-Smith, P. (1992). Neurobiology of an attribute model of memory: Role of prefrontal cortex. In I. Gormezano \& E. A. Wasserman (Eds.), Learning and memory: Behavioral and biological processes (pp. 251-273). Hillsdale, NJ: Erlbaum

Kesner, R. P. \& Novak, J. (1982). Serial position curve in rats: Role of the dorsal hippocampus. Science, 218, 173-174.
Milner, B., Corsi, P., \& Leonard, G. (1991). Frontal-lobe contribution to recency judgements. Neuropsychologia, 29,601-618.

Olton, D. S., Shapiro, M. L., \& Hulse, S. H. (1984). Working memory and serial patterns. In H. L. Roitblat, T. G. Bever, \& H. S. Terrace (Eds.), Animal cognition (pp. 171-181), Hillsdale, NJ: Erlbaum.

Petrides, M. (1991). Learning impairments following excisions of the primate frontal cortex. In H. S. Levin, H. M. Eisenberg, \& A. L. Benton (Eds.), Frontal lobe function and dysfunction (pp. 256-272). Oxford: Oxford University Press.

Shimamura, A. P., Janowsky, J. S., \& SQuire, L. R. (1990). Memory for the temporal order of events in patients with frontal lobe lesions and amnesic patients. Neuropsychologia, 28, 803-813.

SMith, M. L., \& Milner, B. (1988). Estimation of frequency of occurrence of abstract designs after frontal or temporal lobectomy. Neuropsychologia, 26, 297-306.

WINOCUR, G. (1991). Functional dissociation of the hippocampus and prefrontal cortex in learning and memory. Psychobiology, 19, 11-20.

WINOCUR, G. (1992). A comparison of normal old rats and young adult rats with lesions to the hippocampus or prefrontal cortex on a test of matching-to-sample. Neuropsychologia, 30, 769-781.

(Manuscript received January 18, 1993; revision accepted for publication April 14, 1993.) 